\title{
A Evolução da Área de Busca como um Processo de Aprendizagem com base em um Mapeamento Sistemático
}

\author{
Marcelo de O. C. Machado, Cleber Pinelli Teixeira, Sean W. M. Siqueira \\ ${ }^{1}$ Programa de Pós-Graduação em Informática (PPGI) \\ Universidade Federal do Estado do Rio de Janeiro (UNIRIO) \\ Av. Pasteur, 456, Urca - Rio de Janeiro - RJ - Brasil \\ marcelo.machadodedu.unirio.br, \{cleber.pinelli, sean\}@uniriotec.br
}

\begin{abstract}
Search as Learning (SaL) states that search engines must support users through ways to learn during the search process. Previous studies were responsible to propose $S a L$ as a research agenda, carrying different perspectives of investigation and research challenges to be overcome. This paper presents an overview of this research area from an analysis of the publications. We selected 62 papers in which we sought to identify the main authors, the timeline of publications and the main research topics. We believe this research is able to support the development of $S a L$ as a research area by stimulating new proposals.
\end{abstract}

Resumo. A Busca como um Processo de Aprendizagem (BPA) afirma que os mecanismos de buscas devem oferecer suporte aos usuários com meios para aprender durante este processo. Os estudos anteriores foram responsáveis por propor BPA como uma agenda de pesquisa, trazendo perspectivas de investigação e desafios a serem superados. Este artigo apresenta uma visão geral desta área de pesquisa a partir de uma análise das publicações. Foram selecionados 62 trabalhos onde nós buscamos identificar os principais autores, a linha do tempo de publicações e os principais tópicos de pesquisa. Nós acreditamos que esta pesquisa é capaz de auxiliar no desenvolvimento de BPA como uma área de pesquisa, estimulando novas propostas.

\section{Introdução}

A Busca como um Processo de Aprendizagem (BPA) (ou do inglês Search as Learning) é uma área que visa investigar a sinergia entre os processos de busca e aprendizagem [Vakkari, 2016, Rieh et al., 2016]. Ambos os termos referem-se a processos: a aprendizagem sendo um processo, em detrimento de "aprendizado" e a busca, que não se limita apenas a uma tarefa de recuperação da informação, mas se relaciona ao conjunto de atividades desde o momento que se pretende buscar para aprender até o momento em que se considera ter adquirido aprendizado.

Buscar por informações na Web é uma prática quase cotidiana entre os usuários da Internet; e um cenário comum de busca é com intuito de aprendizado. Esse paradigma pró-ativo pode ser classificado como um método de aprendizagem centrado no aprendiz. Assim, o aprendiz se torna responsável pelo seu próprio processo de aprendizagem, transferindo o papel de intermediador do processo de ensino da figura do professor para a interação com as ferramentas de busca. Em BPA acredita-se que o conjunto de tarefas de busca, análise dos resultados e a aprendizagem adquirida durante o processo são os 
VIII Congresso Brasileiro de Informática na Educação (CBIE 2019)

Anais dos Workshops do VIII Congresso Brasileiro de Informática na Educação (WCBIE 2019)

responsáveis pela consolidação de conhecimento. Dentre os objetivos em BPA estão: entender a busca como um processo humano de aprendizagem; mensurar o desempenho de aprendizagem durante a busca e projetar e intervir em sistemas de busca para promover aprendizado [Collins-Thompson et al., 2017].

Alguns estudos serviram para formalizar o relacionamento entre a busca por informação e aprendizagem [Vakkari, 2016, Rieh et al., 2016]. Outros, apresentaram discussões sobre os avanços recentes na área a partir de revisões das publicações [Hansen and Rieh, 2016, Collins-Thompson et al., 2017, Hoppe et al., 2018]. Atualmente, com a crescente investigação sobre o tema, aumenta-se a necessidade de ter uma visão geral da área. Em função disto, e com vistas a alavancar a contribuição nacional e apoiar o desenvolvimento geral dessa agenda de pesquisa selecionamos de maneira sistemática um conjunto de 62 trabalhos na qual foram identificadas as produções anuais, os principais autores, os principais locais de publicação e quais os principais tópicos de pesquisa relacionados. Acreditamos que esta pesquisa apoia novos pesquisadores a compreenderem como a área se configura e a encontrar referências relevantes sobre o tema.

As demais seções estão estruturadas da seguinte maneira: a Seção 2 apresenta os trabalhos relacionados; a Seção 3 apresenta a metodologia utilizada para selecionar e analisar os trabalhos; a Seção 4 discute acerca dos achados relatados; a Seção 5 apresentada as ameaças à validade desta pesquisa e, finalmente, a Seção 6 conclui e apresenta alguns trabalhos futuros. Devido a limitação de espaço, neste trabalho não constam todas as referências dos trabalhos selecionados, entretanto essas referências foram disponibilizadas publicamente ${ }^{1}$ - bem como todos os dados desta pesquisa.

\section{Trabalho Relacionados}

Embora a BPA tenha sido menciona em trabalhos anteriores, as principais pesquisas, alavancaram-se a partir de 2014, através do Searching as Learning Workshop como parte da Information Interaction in Context (IIiX 2014) Conference. À partir desse Workshop as discussões possibilitaram que os trabalhos fossem desenvolvidos. Como resultado, uma visão geral de BPA foi apresentada através da interseção entre busca por informação e fatores de aprendizagem, relatando os principais achados da edição especial da revista de ciência da informação (Journal of Information Science - JIS) de 2016 sobre BPA [Hansen and Rieh, 2016].

Naquele momento Vakkari [2016] mapeou as variáveis dependentes e independentes no contexto de BPA, revelando as principais perspectivas dos experimentos realizados na área. Foram apresentadas as correlações entre busca e aprendizagem e as possíveis linhas de pesquisa a serem exploradas. Rieh et al. [2016] trouxeram um panorama de como a aprendizagem poderá ser melhor identificada através dos históricos e padrões de utilização dos usuários. Foram também elicitados os principais desafios e oportunidades de pesquisa sobre o tema.

Posteriormente, Hoppe et al. [2018] apresentaram os desafios em BPA nas perspectivas de recuperação da informação e psicopedagogia. Através de uma visão crítica, eles argumentam que o impacto do conteúdo multimídia tem sido negligenciado e que as investigações neste sentido deverão ser um dos focos de projetos futuros.

\footnotetext{
${ }^{1}$ https://github.com/marcelomachado/searching_as_learning/tree/master/ systematic-mapping-data
} 
VIII Congresso Brasileiro de Informática na Educação (CBIE 2019)

Anais dos Workshops do VIII Congresso Brasileiro de Informática na Educação (WCBIE 2019)

Em 2017 foi inaugurada uma revista que inclui em seus tópicos de interesse o contexto de BPA: (Information and Learning Sciences - ILS). No artigo editorial de 2019, foram apresentados cronologicamente os principais eventos e iniciativas a fim de encorajar os pesquisadores a investigarem esta área Reynolds et al. [2019] e uma série de reflexões acerca das interseções entre as áreas. Complementarmente, em 2019 houve ainda o I Search Therefore I Learn - ISTIL Workshop at ICWL na Alemanha.

Neste trabalho, buscamos apresentar uma visão geral de BPA a fim de auxiliar os pesquisadores a identificar as principais questões em aberto, servindo para elencar os principais estudos. Possui também o intuito de trazer novas perspectivas de visualização dos avanços promovidos até o momento.

\section{Metodologia}

Este mapeamento sistemático foi organizado de acordo com as principais atividades propostas por Petersen et al. [2015]: planejamento, condução e relato.

\subsection{Planejamento}

Durante a atividade de planejamento foram identificados os objetivos e o protocolo foi definido. O protocolo especifica o método a ser utilizado no mapeamento sistemático com objetivo de reduzir vieses de pesquisa [Holstein et al., 2019] e tornar o processo reprodutível. Esta sub-seção apresenta o protocolo.

\subsubsection{Questões de Pesquisa}

Este mapeamento sistemático visa responder as seguintes questões de pesquisa:

QM1: Quantos estudos sobre SaL foram publicados ao longo dos anos?

QM2: Quem são os autores mais ativos na área?

QM3: Quais locais de publicação são os principais alvos para a produção de pesquisas na área?

QM4: Quais os principais tópicos de pesquisa abordados na área?

\subsubsection{Critérios de Inclusão e de Exclusão}

O conjunto de trabalhos selecionados para as discussões deste trabalho possui o seguinte critério de inclusão: $\mathrm{O}$ artigo apresenta um estudo relacionado à BPA. A seguir são apresentados os critérios de exclusão definidos:

i $\mathrm{O}$ artigo não está em inglês

ii $\mathrm{O}$ artigo tem o conteúdo equivalente a outro artigo mais atual dos mesmos autores

\subsubsection{Formulação da Chave de Busca e Definição das Bases de Artigos}

Para atingir ao objetivo desta pesquisa definimos que os trabalhos selecionados devem se referir explicitamente a BPA. A chave de busca desenvolvida para esta pesquisa contém o principal termo que nomeia a área (Search as Learning) e outros termos que foram 
VIII Congresso Brasileiro de Informática na Educação (CBIE 2019)

Anais dos Workshops do VIII Congresso Brasileiro de Informática na Educação (WCBIE 2019)

identificados como sinônimos a partir da utilização de uma base de trabalhos previamente selecionada pelos autores deste trabalho. A chave de busca final é apresentada a seguir:

("Search as Learning” OR "Searching as Learning” OR "Search as a Learning")

Para a execução dessa chave de busca, foram selecionadas 7 bases de dados: Scopus, Science@Direct, ISI Web of Science, IEEE Digital Library, ACM Digital Library, El Compendex e Google Scholar.

\subsection{Condução do Mapeamento Sistemático}

A execução da chave de busca foi realizada, em cada uma das bases de dados, no mês de setembro de 2019. Ao utilizar a base de dados Science@Direct percebemos que apesar de tentar especificar os termos (utilizando aspas), o sistema de busca dessa base considera os termos com radicais "Search" e "Learn" como disjuntos. Assim qualquer trabalho, que mencione esses termos, mesmo que separadamente, é apresentado como resultado, portanto, foi necessária a inclusão de alguns filtros. Dentre o resultado apresentado (1218 trabalhos) foram excluídas publicações das seguintes áreas: Neurocomputing (40), Neural Networks (27), Technological Forecasting and Social Change (18), Research Policy e IFAC Proceedings Volumes (17). Previamente à exclusão, analisamos uma amostra dos resultados de cada uma dessas áreas. Além disso, apenas trabalhos a partir de 2009 foram considerados, haja vista os resultados das outras bases e a recente discussão do tema. Ao executar a chave de busca na base de dados Google Scholar foram excluídas patentes e citações.

A Figura 1 apresenta os passos e os resultados do processo de condução para seleção dos trabalhos. A partir de um total de 505 trabalhos foram selecionados 62 para análise das questões de pesquisa deste artigo.

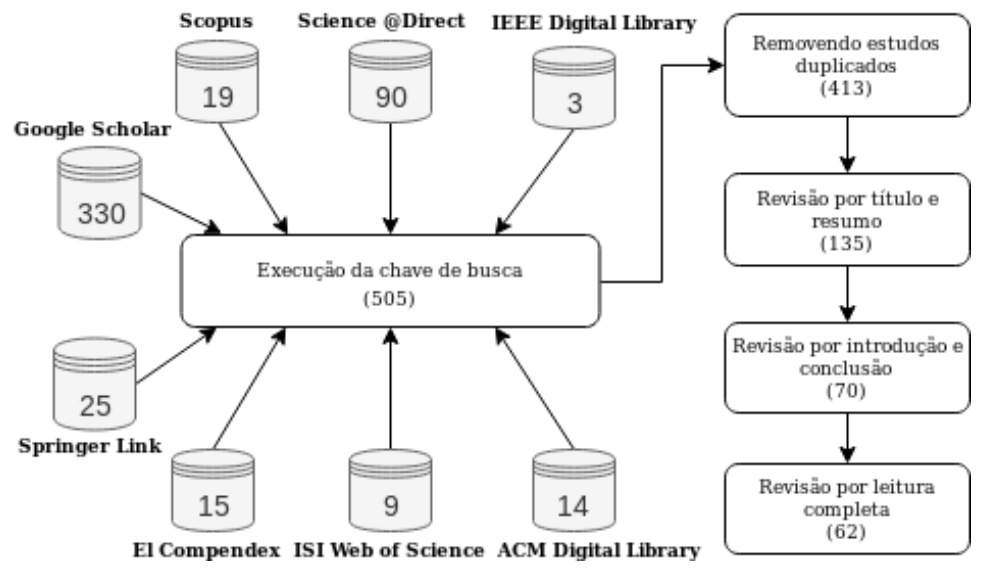

Figura 1. Resultado do processo de condução do mapeamento sistemático.

\subsection{Relatório do Mapeamento}

Esta sub-seção engloba os resultados encontrados a partir da análise dos trabalhos selecionados ao final do processo de condução deste mapeamento sistemático. 
VIII Congresso Brasileiro de Informática na Educação (CBIE 2019)

Anais dos Workshops do VIII Congresso Brasileiro de Informática na Educação (WCBIE 2019)

\subsubsection{QM1: Quantos estudos foram publicados ao longo dos anos?}

Para responder a esta questão de pesquisa foi verificado o número de publicações no decorrer dos anos. A Figura 2 consolida essa informação.

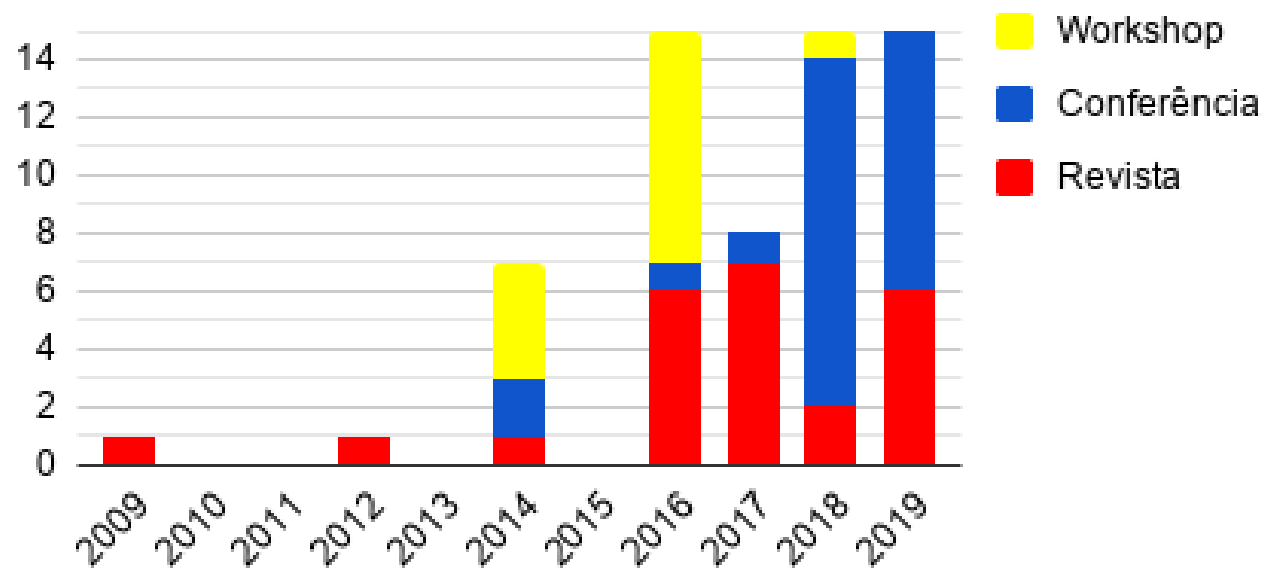

Figura 2. Publicações ao longo dos anos.

\subsubsection{QM2: Quem são os autores mais ativos na área?}

Os principais autores da área e consequentemente os países de atuação foram identificados a partir dos trabalhos selecionados. A Tabela 1 apresenta um recorte dos autores mais ativos na área, considerando autoria e co-autoria. Além disso, a Figura 3 apresenta um recorte de colaboração entre os principais autores da área. Nessa figura, são apresentados apenas sub-grafos que considerem cooperação entre diferentes grupos de autores.

\section{Tabela 1. Amostra dos autores com maior número de publicações (considerando} co-autoria).

\begin{tabular}{lc}
\hline Autores & Artigos \\
\hline Ivana Marenzi (Alemanha), Kevyn Collins-Thompson (EUA), Soo Young Rieh (EUA) & 5 \\
Bernardo Nunes (Brasil), Sean Siqueira (Brasil) & 4 \\
Chirag Shah (EUA), Ion Madrazo Azpiazu (EUA), Marcelo Tibau (Brasil), Maria Sole- & \\
dad Pera (EUA), Nevena Dragovi (EUA), Pertti Vakkari (Finlândia), Peter Holtz (Alema- & 3 \\
nha), Preben Hansen (Suécia), Ran Yu (Alemanha), Rohail Syed (EUA), Stefan Dietze &
\end{tabular}

(Alemanha), Ujwal Gadiraju (Alemanha)

Considerando a quantidade de autores, foi possível identificar os países mais ativos na área: Estados Unidos da América (42), Alemanha (18), Reino Unido (11), China (10), Austrália (7), Holanda (7) e Brasil (5).

\subsubsection{QM3: Quais locais de publicação são os principais alvos para a produção de pesquisas na área?}

Para responder esta questão de pesquisa a Tabela 2 apresenta os principais locais que receberam publicações relacionadas a BPA. Além disso, a Figura 2 apresenta a quantidade de trabalhos no decorrer dos anos considerando o tipo de publicação. 


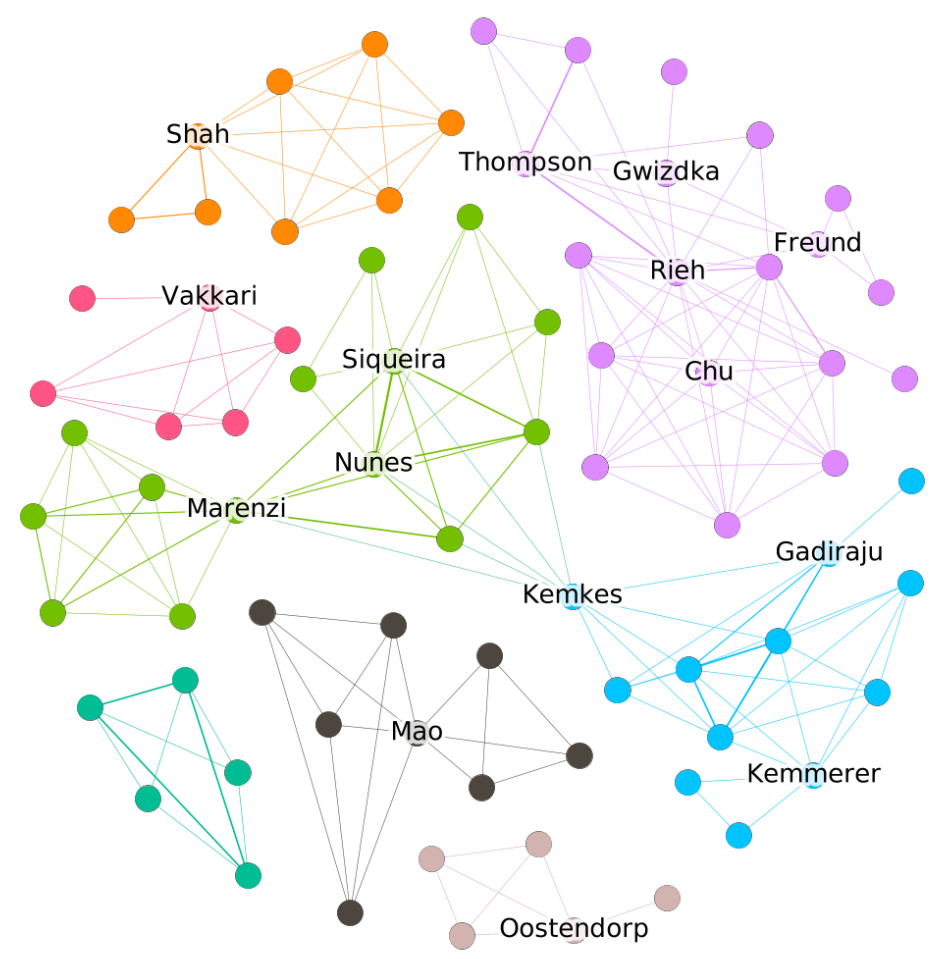

Figura 3. Grafo de colaboração entre os autores.

\subsubsection{Quais os principais tópicos de pesquisa abordados na área?}

A partir da leitura dos trabalhos selecionados foi possível perceber alguns tópicos comumente referenciados, tais quais: variáveis de interação entre usuário e sistema, busca exploratória, teorias de aprendizagem, perfil do usuário, recuperação da informação e representação do domínio de conhecimento. Portanto, para responder a esta questão de pesquisa buscamos classificar os trabalhos de acordo com esses tópicos.

As variáveis de interação entre o usuário e o sistema representam quantitativos de ações do usuário no momento em que está explorando as informações, por exemplo, a quantidade de cliques, o número de páginas visitadas, o tempo de permanência em uma página e a quantidade de reformulações da chave de busca. A busca exploratória envolve as tarefas do usuário no momento em que está investigando os resultados em um processo de busca, portanto, considera a seleção de termos, formulação da consulta, reformulação da consulta, análise dos resultados, entre outros. As teorias de aprendizagem, em Psicologia e em Educação, estão relacionadas a diversos modelos que visam explicar o processo de aprendizagem pelos indivíduos, neste cenário podemos citar teorias de autores tais quais, Jean Piaget, Lev Vygotsky, David Ausubel, entre outros. O perfil do usuário está relacionado com qualquer informação utilizada para representar o modelo do usuário, seja conhecimento prévio, estilo de aprendizagem, habilidades, intenção, entre outros. A recuperação da informação refere-se às intervenções feitas na ferramenta a fim de se adequar ao contexto de aprendizagem, tais como a interação da ferramenta de busca com o usuário (por exemplo, sugestão de termos de consulta e correções do tipo "Você quis dizer...”), reorganização dos resultados das páginas de busca e customização na visualização 
VIII Congresso Brasileiro de Informática na Educação (CBIE 2019)

Anais dos Workshops do VIII Congresso Brasileiro de Informática na Educação (WCBIE 2019)

Tabela 2. Amostra de eventos e periódicos que receberam publicações sobre

BPA.

\begin{tabular}{|c|c|c|c|}
\hline Eventos e periódicos & Ano & Artigos & Total \\
\hline \multirow{3}{*}{ Conference on Human Information Interaction and Retrieval (CHIIR) } & 2016 & 1 & \multirow{3}{*}{9} \\
\hline & 2018 & 3 & \\
\hline & 2019 & 5 & \\
\hline Conference on Research and Development in Information Retrieval (SIGIR) & 2016 & 8 & 8 \\
\hline \multirow{2}{*}{ Information Retrieval Journal } & 2017 & 5 & \multirow{2}{*}{7} \\
\hline & 2019 & 2 & \\
\hline \multirow{3}{*}{ International Conference on Advanced Learning Technologies (ICALT) } & 2017 & 1 & \multirow{3}{*}{4} \\
\hline & 2018 & 1 & \\
\hline & 2019 & 2 & \\
\hline Information Interaction in Context Symposium (IIiX) & 2014 & 4 & 4 \\
\hline Journal of Information Science (JIS) & 2016 & 6 & 6 \\
\hline \multirow{2}{*}{ Information Interaction in Context Symposium (ASIS\&T) } & 2016 & 1 & \multirow{2}{*}{2} \\
\hline & 2018 & 1 & \\
\hline \multirow{2}{*}{ Journal of Information Interaction in Context Symposium (JASIS\&T) } & 2012 & 1 & \multirow{2}{*}{2} \\
\hline & 2017 & 1 & \\
\hline \multirow{2}{*}{ International Conference on Information and Knowledge Management (ACM CIKM) } & 2018 & 1 & \multirow{2}{*}{2} \\
\hline & 2019 & 1 & \\
\hline Information and Learning Sciences (ILS) & 2019 & 2 & 2 \\
\hline \multirow{2}{*}{ Information and Processing Management } & 2009 & 1 & \multirow{2}{*}{2} \\
\hline & 2019 & 1 & \\
\hline
\end{tabular}

da informação. Por fim, a representação do domínio de conhecimento está relacionada a utilização de estruturas de conceitos sobre um domínio de conhecimento e os relacionamentos entre esses conceitos - geralmente aparecem na forma de base de dados ligados ou representações gerais com ontologias.

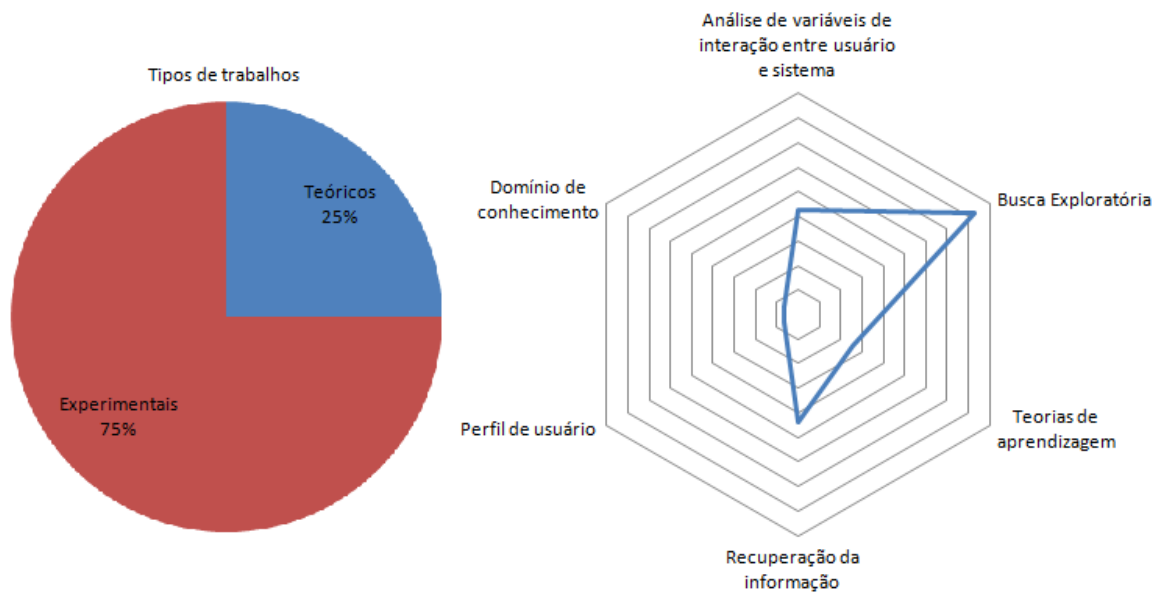

Figura 4. Tipos de trabalhos e análises das áreas mais investigadas nos artigos.

A Figura 4 mostra que a maioria dos estudos retratou aspectos relacionados ao comportamento da busca exploratória (cerca de $83 \%$ dos trabalhos). Referências a variáveis de interação com as fontes de informação foram verificadas em $42 \%$ dos estudos. Aproximadamente $44 \%$ dos trabalho contemplaram a intervenção na ferramenta de busca. Alguns poucos trabalhos apresentaram fundamentação sobre teorias de aprendizagem, aproximando o tema para as perspectivas educacionais e de comportamento humano (cerca de 25\%). A utilização de perfis de usuários e de domínios de conhecimento foi abordada, igualmente, em cerca de $7 \%$ dos trabalhos. 
VIII Congresso Brasileiro de Informática na Educação (CBIE 2019)

Anais dos Workshops do VIII Congresso Brasileiro de Informática na Educação (WCBIE 2019)

\section{Discussões}

A partir da análise temporal de publicações, verificamos que o assunto em questão foi inaugurado por Jansen et al. [2009] a partir de uma estudo empírico acerca do processo de aprendizagem com relação às características únicas de busca de informação. Em seguida em 2012 Vakkari and Huuskonen [2012] analisaram como o esforço de busca está associado ao resultado da busca (respostas às consultas) e ao resultado da tarefa (aprendizado). Apenas em 2014 houve o "boom" de publicações, fomentado pelo primeiro Workshop na área (Searching as Learning Workshop 2014). A partir de 2016 é possível perceber um elevado número de publicações com relação aos anos anteriores, isto se deve a um Workshop de BPA na ACM SIGIR Conference on Research and Development in Information Retrieval e a criação de uma edição especial, sobre a BPA, no Journal of Information Science de 2016. O ano de 2019 juntamente com o ano de 2018 apresentam os maiores números de publicações, todavia, 2019 deve superar 2018, pois novos trabalhos ainda devem ser publicados. Considerando outros idiomas, por exemplo o português, este $1^{\circ}$ Workshop de Busca como um Processo de Aprendizagem também irá apoiar o fortalecimento de publicações em 2019, por fim, demonstrando o crescimento da área.

Com base no relatório da segunda questão de pesquisa, reconhecemos os principais autores da área. A Figura 3 apresenta o relacionamento de co-autoria entre esses autores, demonstrando forte cooperação entre os grupos de pesquisa, destacando-se as pesquisadoras Soo Young Rieh e Ivana Marenzi e o pesquisador Philipp Kemkes que são os principais pontos de interseção entre grupos. A cooperação entre os grupos favorece a formalização e disseminação da área. Além disso, o relatório apontou os principais países identificados a partir do grupo de pesquisa dos autores. No Brasil, os principais autores apresentam colaboração de pesquisadores da Alemanha, Austrália, Itália e Colômbia.

No relatório da terceira questão de pesquisa foram apontados os principais locais de publicação. Este resultado, juntamente com a Figura 2, revela uma área em fase inicial. Todavia, nos últimos anos o número de publicação em revistas vem aumentando demonstrando que alguns resultados já podem ser observados nesta área de pesquisa. Percebemos ainda a relação entre a área de BPA com outros campos de pesquisa. Apesar disto, as conferências que receberam maior quantidade de publicações foram CHIIR e SIGIR e a revista com mais publicações foi Information Retrieval Journal. Portanto, isso evidencia o relacionamento de BPA com a área de Recuperação da Informação, cujo viés de investigação tem sido muito mais quantitativo, carecendo de fundamentações relacionadas ao comportamento humano, sobretudo à aprendizagem.

Por fim, a partir do relatório da quarta questão de pesquisa foi possível reconhecer os principais tópicos de pesquisa, ressaltando as variáveis de interação entre o usuário e o sistema, a busca exploratória e a recuperação da informação. Diferentemente de outras áreas relacionadas a aprendizagem em ambientes de educação online o perfil do aluno e a representação do domínio de conhecimento foram explorados em poucos trabalhos demonstrando que inicialmente a área de BPA não tem um foco na personalização direta do conteúdo. Jansen et al. [2009] indicam que no geral, uma teoria da aprendizagem pode descrever melhor o processo de busca de informações do que os paradigmas comuns de tomada de decisão ou solução de problemas. Entretanto, notamos que apesar da área de Aprendizagem estar no núcleo desta agenda de pesquisa poucos trabalhos demonstraram de maneira explícita a fundamentação em teorias ou paradigmas de aprendizagem. Desta 
VIII Congresso Brasileiro de Informática na Educação (CBIE 2019)

Anais dos Workshops do VIII Congresso Brasileiro de Informática na Educação (WCBIE 2019)

maneira, entendemos que as comunidades da Educação e Psicologia precisam tomar conhecimento sobre o tema a fim de contribuir para uma interpretação mais adequada do que entende-se sobre aprendizagem neste contexto de busca.

\section{Ameaças à Validade}

Este mapeamento sistemático da literatura teve como objetivo apresentar uma visão geral desta agenda de pesquisa. No entanto, como qualquer método, existem ameaças à sua validade e limitações. Os resultados deste estudo podem ter sido influenciados por certas limitações incontroláveis. Foram cobertos 62 estudos, por outro lado foram desconsiderados aqueles não escritos em inglês ou que não tenham sido rigorosamente revisados por pares, ameaçando a validade das conclusões.

Pode haver um viés em relação ao número de pesquisadores que selecionaram os trabalhos. Além de revisar o processo geral, com o objetivo de mitigar essa ameaça à validade, mais de um pesquisador conseguiu reproduzir esse processo para reduzir a possibilidade de viés. A inclusão ou exclusão de trabalhos pode ser subjetiva ou propensa a erros. No entanto, buscou-se atenuar essa ameaça fazendo com que duas pessoas verificassem a inclusão/exclusão de artigos, alcançando um consenso.

Os critérios de pesquisa sistemática também podem estar sujeitos a críticas, ameaçando a validade do construto. A chave de busca pode não conter todas as palavraschave relevantes, causando a perda de alguns estudos valiosos e erros podem ser inseridos na definição do protocolo. Para atenuar isso, a chave de busca foi avaliada usando um conjunto de artigos previamente selecionados.

Em relação à validade externa, é importante demonstrar repetibilidade. Assim, todos os dados desta pesquisa foram disponibilizados publicamente.

\section{Conclusões e Trabalhos Futuros}

Este estudo trouxe uma visão geral sobre o panorama da pesquisa em BPA e uma análise baseada na evolução das publicações da área. A condução do protocolo especificado, permitiu a seleção de 62 trabalhos que tratam da sinergia entre os processos de busca e aprendizagem. Todos os dados relacionados a esta condução foram disponibilizados para reprodução do processo e para permitir discussões sobre a área.

Sobre os achados da pesquisa, identificamos os principais autores e países envolvidos na área, os principais locais de publicação e os principais nichos de pesquisa. Evidenciamos que BPA é uma área em ascensão com seus principais trabalhos a partir de 2016. Dentre as discussões apresentadas, destacamos que, apesar da área envolver a Aprendizagem em seu escopo, poucos trabalhos se fundamentaram explicitamente em teorias de aprendizagem.

Em trabalhos futuros, vislumbramos a utilização da técnica metodológica de bola de neve, para incorporar trabalhos que estejam relacionados à BPA mesmo que de maneira indireta. Também aspiramos a criação de um modelo de BPA objetivando a formalização da área a partir dos tópicos de pesquisa elencados neste trabalho. Ainda, pretendemos fazer uma análise dos trabalhos dessa pesquisa buscando compreender que tipos de teorias e/ou paradigmas de aprendizagem estão sendo utilizados (mesmo que de maneira implícita). 
VIII Congresso Brasileiro de Informática na Educação (CBIE 2019)

Anais dos Workshops do VIII Congresso Brasileiro de Informática na Educação (WCBIE 2019)

Dada a importância do tema visualizamos como prognóstico um maior amadurecimento da área nos próximos anos. Acreditamos que alguns aspectos precisam ser devidamente formalizados a fim de auxiliar os pesquisadores a contribuir para os avanços em BPA no desenvolvimento de ferramentas, métodos e processos. Esta formalização deverá tanger aspectos interdisciplinares que nos permita ter uma visão sistêmica a fim de promover mais teorizações, experimentações e intervenções neste contexto de BPA.

\section{Agradecimentos}

Este estudo foi parcialmente financiado pelo Conselho Nacional de Desenvolvimento Científico e Tecnológico (CNPq), projeto: Searching as Learning: a busca de informação como ferramenta para a aprendizagem, processo $\mathrm{n}^{\mathrm{o}}$ : 315374/2018-7. E realizado com apoio da Coordenação de Aperfeiçoamento de Pessoal de Nível Superior - Brasil (CAPES) - Código de Financiamento 001.

\section{Referências}

Kevyn Collins-Thompson, Preben Hansen, and Claudia Hauff. Search as Learning (Dagstuhl Seminar 17092). Dagstuhl Reports, 7(2):135-162, $2017 . \quad$ ISSN 21925283. doi: 10.4230/DagRep.7.2.135. URL http://drops.dagstuhl.de/ opus/volltexte/2017/7357.

Preben Hansen and Soo Young Rieh. Recent advances on searching as learning: An introduction to the special issue, 2016.

Kenneth Holstein, Bruce M McLaren, and Vincent Aleven. Designing for complementarity: Teacher and student needs for orchestration support in ai-enhanced classrooms. In International Conference on Artificial Intelligence in Education, pages 157-171. Springer, 2019.

Anett Hoppe, Peter Holtz, Yvonne Kammerer, Ran Yu, Stefan Dietze, and Ralph Ewerth. Current challenges for studying search as learning processes. 2018.

Bernard J Jansen, Danielle Booth, and Brian Smith. Using the taxonomy of cognitive learning to model online searching. Information Processing \& Management, 45(6): 643-663, 2009.

Kai Petersen, Sairam Vakkalanka, and Ludwik Kuzniarz. Guidelines for conducting systematic mapping studies in software engineering: An update. Information and Software Technology, 64:1-18, 2015.

Rebecca Reynolds, June Ahn, Simon Shum, Preben Hansen, Caroline Haythornthwaite, Hong Huang, Eric Meyers, and Soo Young Rieh. Inaugural issue perspectives on information and learning sciences as an integral scholarly nexus. pages 2-18, 01 2019. doi: 10.1108/ILS-01-2019-138.

Soo Young Rieh, Kevyn Collins-Thompson, Preben Hansen, and Hye-Jung Lee. Towards searching as a learning process: A review of current perspectives and future directions. Journal of Information Science, 42(1):19-34, 2016. doi: 10.1177/0165551515615841.

Pertti Vakkari. Searching as learning: A systematization based on literature. Journal of Information Science, 42(1):7-18, 2016.

Pertti Vakkari and Saila Huuskonen. Search effort degrades search output but improves task outcome. Journal of the American Society for Information Science and Technology, 63(4):657-670, 2012. 